\title{
Study of energetic particle physics with advanced ECEI system on the HL-2A tokamak
}

\author{
Zhongbing Shi ${ }^{1, *}$, Min Jiang ${ }^{1}$, Liming Yu ${ }^{1}$, Wei Chen ${ }^{1}$, Peiwan Shi ${ }^{1}$, Wulyu Zhong ${ }^{1}$, Zengchen Yang ${ }^{1,2}$, Boyu Zhang ${ }^{1}$, \\ Xiaoquan $\mathrm{Ji}^{1}$, Yonggao $\mathrm{Li}^{1}$, Yan Zhou ${ }^{1}$, Shaodong Song ${ }^{1}$, Mei Huang ${ }^{1}$, Xianming Song ${ }^{1}$, Jiaxuan $\mathrm{Li}^{1}$, Baoshan $\mathrm{Yuan}^{1}$, \\ Bingzhong Fu${ }^{1}$, Zetian Liu ${ }^{1}$, Xuantong Ding ${ }^{1}$, Yuhong Xu${ }^{1}$, Qingwei Yang ${ }^{1}$, and Xuru Duan ${ }^{1}$
}

${ }^{1}$ Southwestern Institute of Physics, Chengdu, 610041, China

${ }^{2}$ Xihua University, Chengdu, 610039, China

\begin{abstract}
Understanding the physics of energetic particles (EP) is crucial for the burning plasmas in next generation fusion devices such as ITER. In this work, three types of internal kink modes (a saturated internal kink mode (SK), a resonant internal kink mode (RK), and a double e-fishbone) excited by energetic particles in the low density discharges during ECRH/ECCD heating have been studied by the newly developed 24 (poloidal $) \times 16($ radial $)=384$ channel ECEI system on the HL-2A tokamak. The SK and RK rotate in the electron diamagnetic direction poloidally and are destabilized by the energetic trapped electrons. The SK is destabilized in the case of $q_{\min }>1$, while the RK is destabilized in the case of $q_{\min }<1$. The double e-fishbone, which has two $m / n=1 / 1$ modes propagating in the opposite directions poloidally, has been observed during plasma current ramp-up with counter-ECCD. Strong thermal transfer and mode coupling between the two $m / n=1 / 1$ modes have been studied.
\end{abstract}

\section{Introduction}

The MHD activities induced by energetic particles (EP), which may cause severe confinement degradation in the burning plasma devices such as ITER and future fusion reactor, have been concerned by many scientists [1]. One of those is the fishbone instability, which was first observed in 1983 in PDX tokamak with nearly perpendicular neutral beam injection (NBI) heating [2]. Then, various types of fishbones, have been observed in many fusion devices [3-8] with different heating, such as electron cyclotron resonance heating and current drive (ECRH/ECCD), lower hybrid current drive (LHCD), and/or ion cyclotron resonance heating (ICRH). The fishbone mode is also called resonant internal kink mode (RK). It is widely accepted that the structure of the RK is a $m / n=1 / 1$ mode, which is destabilized by energetic trapped particles via processional resonance [9]. These phenomena have been explained by resonance between energetic particles and internal kink mode (IKM) at $q=1$ resonant surface $[9,10]$. An interesting property of RK is their nonlinear dynamics including robust burst, frequency chirping and jumping, which is associated with the redistribution and the losses of energetic particles. The saturated fishbone mode is also called saturated internal kink mode (SK), which originates from its absence of the $q=1$ resonant surface (in other words: $q_{\min }>1$ ) [11]. The SK has characteristics of saturated amplitude, slow change or constant frequency, and long lasting time. Therefore, the SK is also call long lived mode or long lasting mode (LLM) in some literatures.
The SKs have been observed and investigated on MAST [12], NSTX [13] and HL-2A [14,15]. When these modes appear, there are strong damping of the toroidal rotation, degradation of stored energy and significant losses of fast particles. Both RK and SK are the core localized internal-kink-like modes, with the mode numbers of $\mathrm{m} / \mathrm{n}$ $=1 / 1$. Previous studies suggested that, in a weak/reversed shear $q$ profile configuration with a suprathermal electron population, the e-fishbone may be induced in an electron dominated heating plasma.

In this work, three types of internal kink modes excited by energetic particles have been studied by the newly developed electron cyclotron emission imaging (ECEI) system with the ECRH/ECCD heating on the HL-2A tokamak. Significant results are as follows: Both the $\mathrm{SK}$ and $\mathrm{RK}$, rotating in the electron diamagnetic direction poloidally, are destabilized by the energetic trapped electrons. The electron SK (e-SK) is destabilized in the case of $q_{\min }>1$, while the electron RK (e-RK) is destabilized in the case of $q_{\min }<1$. A double e-fishbone has been observed for the first time experimentally. It has two $m / n=1 / 1$ modes which propagate in the opposite directions poloidally. The paper is organized as follows: the arrangement of the experiment is presented in section 2. The key diagnostic, 24(poloidal) $x$ $16($ radial $)=384$ channel ECEI system, is described in section 3 . The main experimental results, including the e-RK, e-SK, and double e-fishbone have been shown in section 4 and 5 , respectively. The summary is given in section 6 .

\footnotetext{
*Corresponding author: shizb@swip.ac.cn
} 


\section{Arrangement of the experiment}

The HL-2A device is a medium-sized circular tokamak with a major radius $R_{a x}=165 \mathrm{~cm}$, minor radius $a=40$ $\mathrm{cm}$ and a closed diverteor, which can be operated in either a limiter configuration or a single null (SN) divertor configuration [16]. The achieved operation parameters are plasma current of $I_{p}=450 \mathrm{kA}$, toroidal magnetic field of $B_{t}=2.7$ Tesla, electron and ion temperature of $5 \mathrm{keV}$ and $2.8 \mathrm{keV}$, respectively, and a line averaged plasma density of $\sim 8 \times 10^{19} \mathrm{~m}^{-3}$. The vessel is covered with the carbon fiber compound (CFC) and is routinely siliconized. The available auxiliary heating and current drive power includes $2 \mathrm{MW} \mathrm{NBI}$ (ion energy: 45keV), 3 MW ECRH (six gyrotrons, 68 $\mathrm{GHz}$ ) and 1MW LHCD (two klystrons: $2.45 \mathrm{GHz}$ ). The ECRH power with $\mathrm{O}$ mode (selected fundamental harmonic) or X-mode (second harmonic) is injected from the low field side (LFS). The transmission efficiency can achieve 0.9. The launching angle of ECRH antennas can vary from -30 to 30 degree, toroidally. This enable us to change the driving effciency and the driving direction.

To study the instability of energetic particles, the experiments are arranged at low density condition (about $0.5 \times 10^{19} \mathrm{~m}^{-3}$ ) with high auxiliary heating power, in order to achieve high temperature but low collisionality operation regime. The previous work suggested that the internal kink mode, i.e. fishbone, can be easily excited in a weak/negative magnetic shear configuration [14, 15]. The electron cyclotron current drive is used to control the magnetic configuration. Four gyrotrons are used in this experiment. Among them, two gyrotrons are used for heating and the other two are used for electron cyclotron current drive (ECCD).

The key diagnostics for this experiment are the soft $\mathrm{X}$-ray array, the far-infrared (FIR) laser interferometer, the X-mode reflectometer, the ECE and ECEI diagnostic. The soft X-ray intensity is sensitive to the electron temperature, density and effective charge, and is given by $I_{s x r} \sim n_{e}^{2} T_{e}^{a} f\left(Z_{e f f}\right)$ [17]. The exponent $a$ is an increasing function of the absorber foil thickness; its value is usually between 2 and 3. Therefore, small perturbations of $n_{e}, T_{e}$ and $Z_{\text {eff }}$ can easily be detected. The multichannel far-infrared interferometer-polarimeter is used to measure the line averaged electron density and the Faraday rotation angle [18]. The $\mathrm{Q} / \mathrm{V}$ band $\mathrm{X}$-mode reflectometer is used to measure the density profile with the temporal resolution of $\sim 0.02 \mathrm{~ms}$, in the case of $B_{t} \sim$ 1.3 Tesla [19]. The 56-channel ECE with spatial resolution about $1 \mathrm{~cm}$ is used to measure the temperature profile and its evolution across the whole plasma area [20]. The absolute value of the electron temperature is obtained by cross calibration with temperature measured by the core Thomson Scattering system. It should be noted that the FIR laser interferometer and soft-x-ray record central chord line-averaged signals and chordintegrated signals, respectively, whereas the X-mode reflectometer and ECE/ECEI diagnostic record local parameters of electron density and temperature in concert with the reduced toroidal magnetic field, which decreases monotonically with radius. Due to its local measurement and high spatial resolution, the ECE/ECEI diagnostics are also used to determine the accurate locations and the mode structures of the MHD instabilities. The local temperature measurement from ECE/ECEI diagnostic is determined by the optical thickness, which has been discussed in many literatures $[20,21]$. In this experiment, the electron densities of the analyzed discharges are about $0.5 \times 10^{19} \mathrm{~m}^{-3}$. This will cause optical thin at the plasma edge. But at the plasma center, inside $r / a=0.5$, the calculated optical thickness is tau $>2$ in our analyzed discharges. Since the observed internal kink modes are located inside $r / a=0.5$ in this work, the ECE/ECEI measurements are reliable.

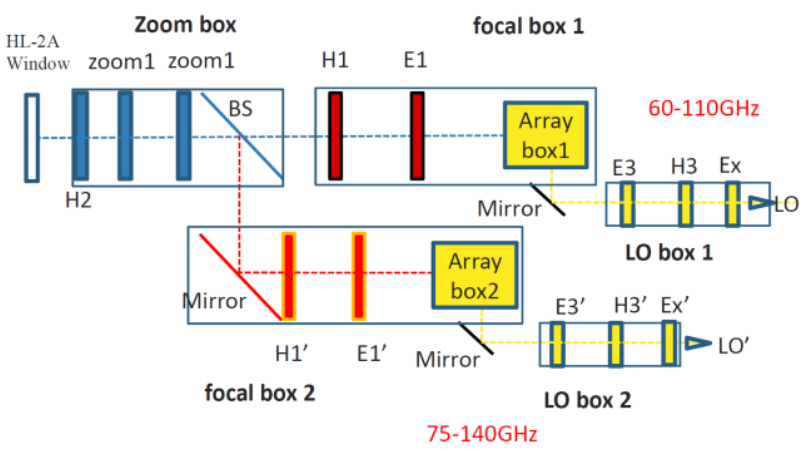

Fig.1. Schematic diagram of the dual-array ECEI optical system on HL-2A.

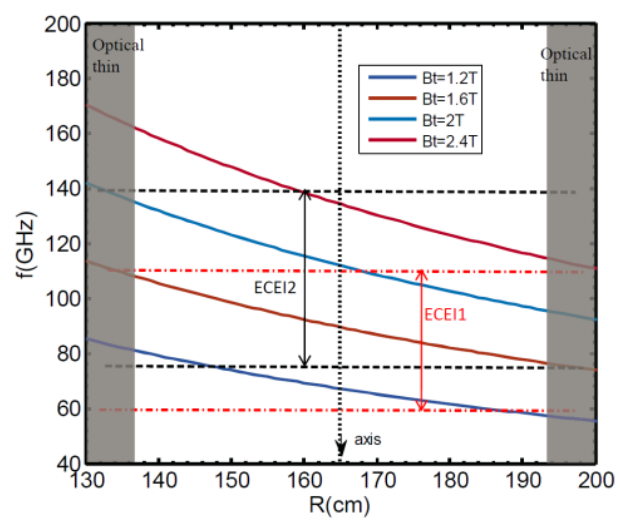

Fig.2. Frequency range of the $2^{\text {nd }}$ harmonic X-mode ECE radiation as a function of HL-2A major radius. The shaded area denotes the third harmonic overlap region, where the optical thickness is thin.

\section{4 channel ECEl system}

The electron cyclotron emission imaging (ECEI) system on the HL-2A tokamak has been developed to visualize the two-dimensional (2D) temperature fluctuations. The ECEI system is comprised of a quasi-optical system, a front-end 24 channel heterodyne imaging array with a tunable radio frequency from 60 to $140 \mathrm{GHz}$, and a set of back-end ECEI electronics that together generate $24($ poloidal $) \times 16($ radial $)=384$ channel images of the $2 \mathrm{D}$ temperature fluctuations.

Figure 1 shows the schematic diagram of the ECEI optical system. It is a dual array ECEI system. The optical system consists of one zoom optics box, two focal optics boxes and two local oscillator (LO) optics boxes. The zoom optics box has two E-plane zoom 
lenses, and the field of view can be adjusted easily by changing the position of the zoom lenses. The ECE signal from plasma is captured by the zoom lenses. Then, the signal is power divided by a $3 \mathrm{~dB}$ beam splitter (BS). One part of the beam transmits through the BS to the focal optics box 1 . The beam goes through the focal lenses $\mathrm{H} 1$ and E1, arrives at the first antenna array in the focal optics box 1 , and is then down-converted to the intermediate frequency (IF) by the microwave mixers in the first antenna array with the LO beam from the first LO optics. Another part of the beam is reflected by the $\mathrm{BS}$, then reflected by a large mirror, goes through the focal lenses $\mathrm{H} 1$ and $\mathrm{E} 1$ in the focal optics box 2, arrives at the second antenna array, and is then down-converted to the IF by the microwave mixers in the second antenna array with the LO beam from the second LO optics.

The two LO and focal optics have different working frequencies. One works at $60-110 \mathrm{GHz}$ and the other one works at 75-140 GHz. Figure 2 shows the frequency range of the $2^{\text {nd }}$ harmonic X-mode ECE radiation with $B_{t}$ $=1.2-2.4$ Tesla as a function of HL-2A major radius. The shaded area in the figure marks the region which is overlapped with the third harmonic ECE $(R<137 \mathrm{~cm}$ and $R>193 \mathrm{~cm}$ ) and the optical thickness is thin. Because the two LO frequencies are independent, the measurement region can be flexibly adjusted according to the physical requirement, by changing the working frequency under different toroidal magnetic field operations. The size of the working pattern, or the vertical resolution, is determined by the zoom optics. By changing the position of the zoom lenses, the optical system can work at between wide and narrow zoom. The wide zoom has a vertical resolution of $2.3 \mathrm{~cm}$. It is designed to provide a large viewing coverage of the plasma with a vertical height of $55 \mathrm{~cm}$. This is very useful for the large-scale MHD structure measurement. The narrow zoom has a vertical resolution of $1.7 \mathrm{~cm}$. It is designed to view the delicate structures of the smallscale MHD in the plasma with a vertical height of $40 \mathrm{~cm}$. Details of the system calibration can be found in reference [22].

\section{Resonant internal kink mode and saturated internal kink mode}

The internal kink mode induced by energetic electrons can be excited easily during high power on-axis ECRH in the low density plasmas on HL-2A [7,14,23]. The magnetic shear can be controlled by the current drive. The current driving direction of ECCD can change the magnetic shear, which affect the excitation of the internal kink mode. In order to clarify the effect of heating and driving direction of EC wave on internal kink mode, two discharges with opposite ECCD driving directions have been studied. As shown in Fig.3(a) and (c), they have same plasma current, density and ECRH/ECCD power, which are $133 \mathrm{kA}, 0.5 \times 10^{19} \mathrm{~m}^{-3}$ and $1 \mathrm{MW}$, respectively. Figure 3 (b) and (d) show spectrograms of the central soft X-ray signals. The e-RK is found in condition of counter-ECCD/ECRH. The frequency of the e-RK decreases from 15 to $5 \mathrm{kHz}$ and accompanied with sawtooth crashes. The e-SK is found in the condition of co-ECCD/ECRH. Unlike conventional RK with frequency chirping or jumping behaviors $[7,14]$, its frequency is nearly unchanged. This suggests that this mode is saturated. It may closely relate to the weakly reversed shear due to ECCD [24,25].
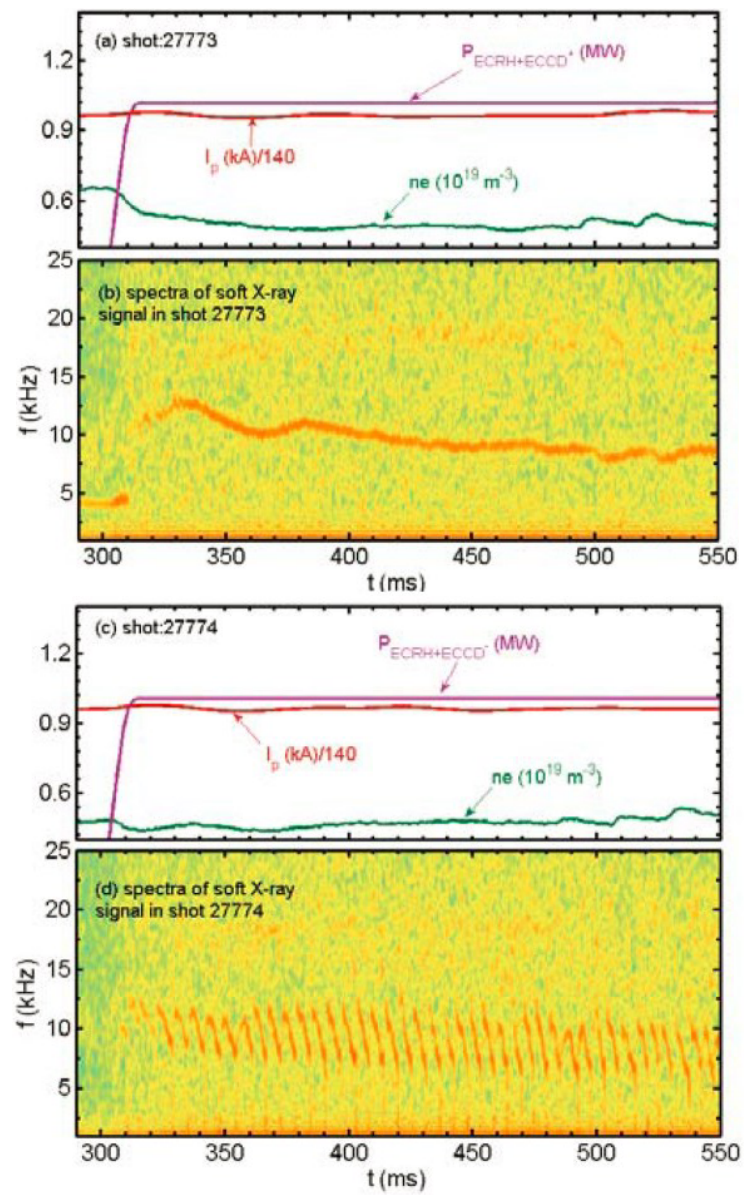

Fig. 3. Effects of current driving directions of ECCD on the internal kink mode. $(\mathrm{a}, \mathrm{c})$ discharge parameters, and $(\mathrm{b}, \mathrm{d})$ spectrograms of soft X-ray signals in shot 27773 and 27774.

Figure 4 shows time evolution of the mode structures of the e-RK and e-SK measured by the ECEI system. There are two obvious islands, a hot and a cold one, and the mode locates almost within a $r=10 \mathrm{~cm}$ circle (the $q=1$ surface). So the mode numbers are $\mathrm{m} / \mathrm{n}$ $=1 / 1$. The intensity of e-SK is higher than that of e-RK. This suggested that the SK might cause a strong damping of the toroidal rotation, degradation of stored energy and significant fast particle loss. Though the opposite current driving directions of ECCD for e-RK and e-SK, their propagating directions are in the anticlockwise direction, which is the electron diamagnetic direction, poloidaly.

Figure 5 shows the q-profiles during e-RK and eSK modes in shot 27773 and 27774. They are calculated by the current filament method and modified by Faraday rotation measured by FIR laser polarimeter interferometer. The negative q-profiles are formed by the high power ECRH for both conditions. The $q_{\min }$ is a slight bigger than unity with co-ECCD at $\mathrm{t}=505 \mathrm{~ms}$ in shot 27773 , while the $q_{\min }$ is a bit smaller than unity with 
counter-ECCD at $\mathrm{t}=505 \mathrm{~ms}$ in shot 27774 . There are obvious sawtooth crashes in shot 27774 (RK condition) from soft X-ray sig nals, while there are no sawtooth in shot 27773 (SK condition). The signals from soft X-ray arrays also indicate that the $q_{\min }$ is a little bigger than unity in shot 27773 , while in shot 27774 , the $q_{\min }$ is smaller than unity. This suggests that there is a $q_{\text {min }}$ condition for the destabilization of SK. The SK is destabilized in the case of $q_{\min }>1$, while the RK is destabilized in the case of $q_{\min }<1$.

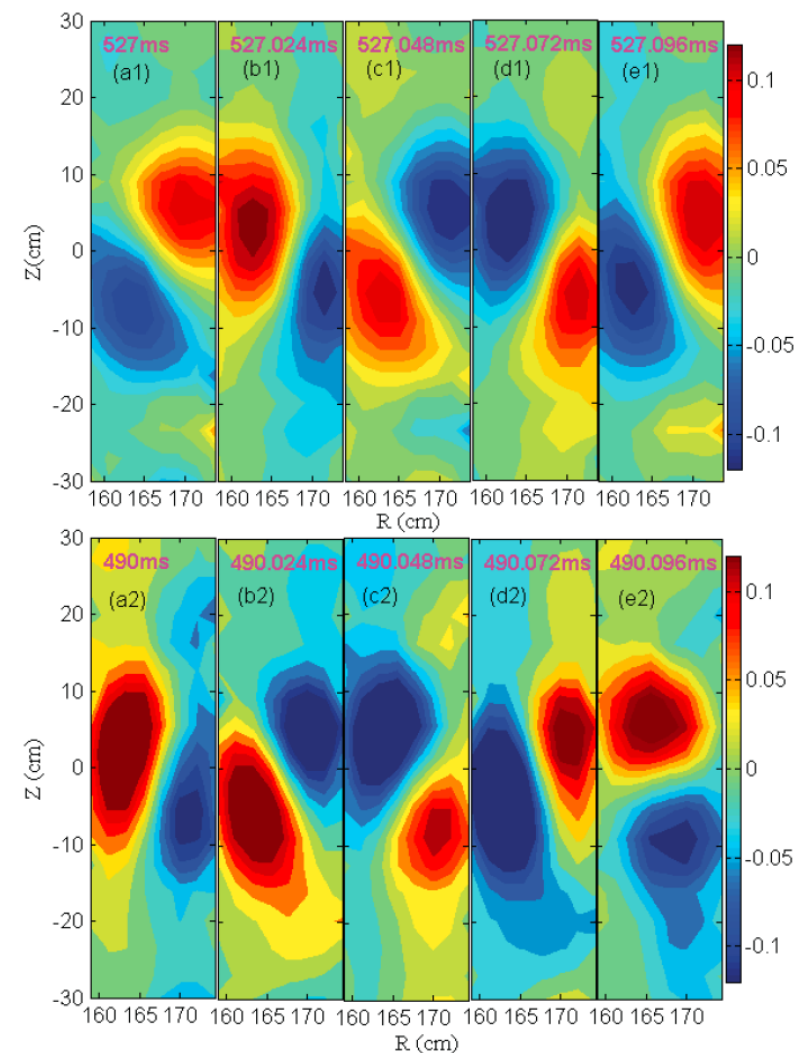

Fig.4. Mode structure evolutions of e-RK (a1,b1,c1,d1,e1) in shot: 27774 and e-SK (a2,b2,c2,d2,e2) in shot: 27773 measured by the ECEI system.

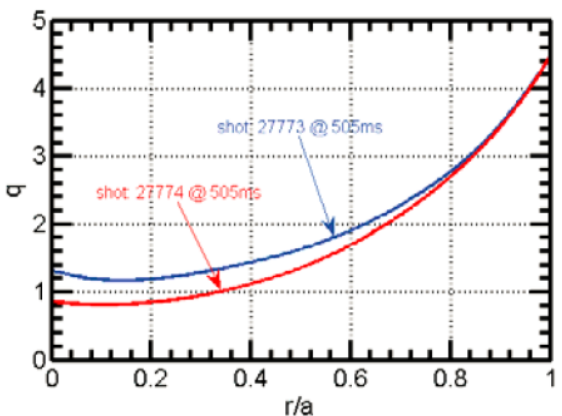

Fig. 5. q-profiles during e-RK and e-SK in shot 27773 and 27774 at $\mathrm{t}=505 \mathrm{~ms}$.

\section{Double e-fishbone}

With counter-ECCD injection during the plasma current ramp-up, the double structure of e-fishbones have been observed [26]. The typical discharge with double efishbone instability is shown in Fig.6. Figure 6(a) shows the plasma current, which ramps up from $146 \mathrm{kA}$ at 540 $\mathrm{ms}$ to $165 \mathrm{kA}$ at $590 \mathrm{~ms}$. The counter-ECCD/ECRH with power of $850 \mathrm{~kW}$ was injected into plasma during current ramp up. As shown in Fig.6(c) and (d), evident electron temperature fluctuation appears at $548 \mathrm{~ms}$, and its amplitude gradually increases and frequency gradually drops from $20 \mathrm{kHz}$ to $10 \mathrm{kHz}$ until $563 \mathrm{~ms}$. The mode disappears at $563 \mathrm{~ms}$ due to the gas puffing, which causes the increase of the density at this time. The decrease of the mode frequency may be due to the variation of the $q_{\min }$. After about $5 \mathrm{~ms}$, when the density back to about $0.5 \times 10^{19} \mathrm{~m}^{-3}$, the mode appears again, and the mode frequency nearly maintains at about $7.5 \mathrm{kHz}$ from $568 \mathrm{~ms}$ to $579 \mathrm{~ms}$.

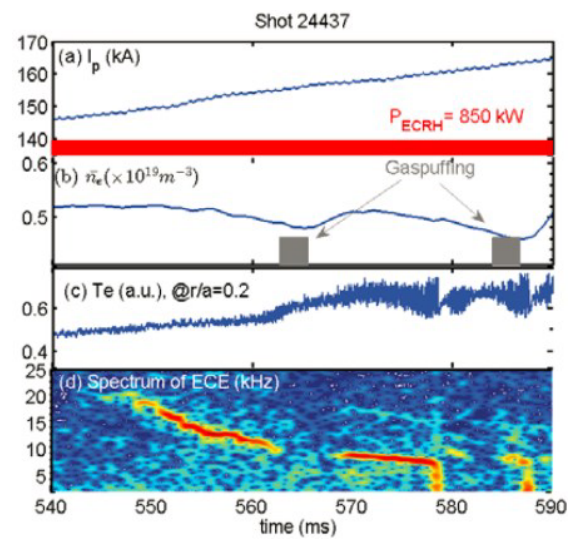

Fig.6. Temporal evolution of (a) plasma current and ECRH power (b) line-averaged electron density and gas puffing pulses, (c)ECE signal at $\mathrm{r} / \mathrm{a}=0.2$, (d) Frequency spectrum of central ECE signal.

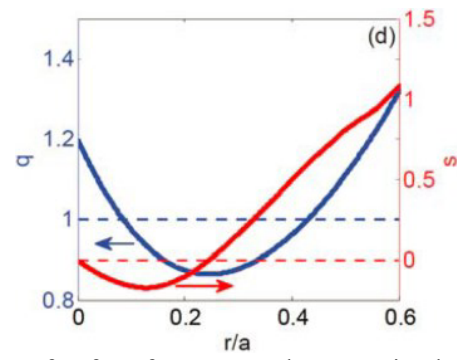

Fig.7. Profiles of safety factor $q$ and magnetic shear $s$ in shot 24437 at $570 \mathrm{~ms}$.

The deposition of the ECRH and amplitude of driven current are simulated through a linear ray tracing code TORAY-GA [27], which computes the trajectories of Gaussian rays representing ECRH microwave beams using cold plasma dispersion relation. The ECRH power is almost deposited inside $r / a=0.35$, and peaks at $r / a=$ 0.2 . The driven current is about $13.6 \mathrm{kA}$. Figure 7 shows the profiles of the safety factor $q$ and the magnetic shear $s$ at $570 \mathrm{~ms}$ in shot 24437. The q-profile is constructed by the current filament method, where the plasma equilibrium configurations are fitted by using the internal magnetic field measurements of Faraday rotation effect, and external magnetic field measurements with magnetic probes and flux loops. The magnetic shear $s$ is given by $s=r d q / q d r$. We can find that there are two $q=$ 1 surfaces. The locations of the two $q=1$ surfaces are $r / a=0.12$ and $r / a=0.42$ respectively. It is observed that 
there is weak negative shear configuration in the core plasma.
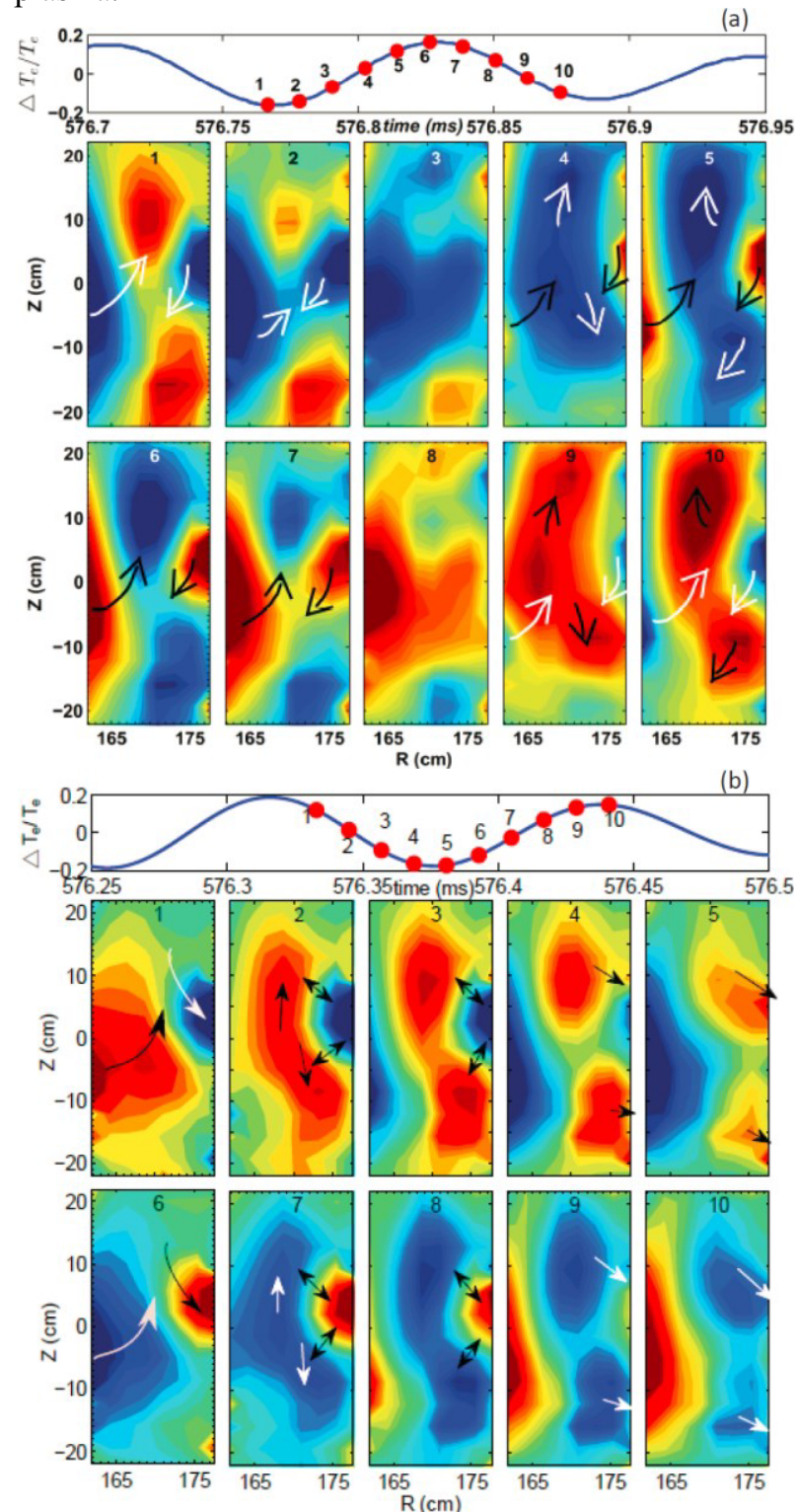

Fig.8. Temporal frames of the double e-fishbone (a) when the hot-hot (cold-cold) spots of the two modes collide with each other, (b) when the hot (cold) spot of one mode approaches the cold (hot) spot of the other mode. The white and black arrows indicate the moving trend of the cold and hot spots, respectively. The double arrow means the interaction between the two modes. When hot spots meet with cold spots, the enhanced outwards transport was observed.

The temporal evolutions of the $2 \mathrm{D}$ structures of the double e-fishbone have been observed by ECEI system. It has two $m / n=1 / 1$ modes. The locations of the two modes are found to be $R=170 \mathrm{~cm}(r / a=0.15)$ and $R=$ $178 \mathrm{~cm}(r / a=0.41)$, which are consistent with that of the two $q=1$ surfaces shown in Fig.7. As shown in Fig.8, the two $m / n=1 / 1$ modes with opposite propagation directions have been found clearly: the inner mode propagates in the electron diamagnetic drift direction and the outer mode propagates in the ion diamagnetic direction. In these frames, we can see that there are two cold spots and two hot spots with different propagation direction respectively. The white and black arrows indicate the moving trend of the cold and hot spots, respectively. As seen in Fig. 8(a), when the two cold (or hot) spots are far away, they can be clearly recognized to rotate in the opposite direction. When they are close to each other, the two clusters of cold (or hot) spots seems to merge together and then becomes elongated. Afterwards, the elongated cold spots seems to split into two parts, and continue to move in the opposite directions. In this dynamics process, no enhanced transport has been observed. However, as seen in Fig. $8(b)$, when the inner hot spot meets the cold spot of the outer mode, the obvious heat energy transition has be observed between the hot and cold spot. The double arrow in this figure denotes the interaction between the two modes. This process can be observed clearly with a cartoon or movie. The heat transfer seems to begin at the largest gradient of the temperature, where the temperature decreases firstly, then the hot spot splits into two parts and the two heat parts transfer outward quickly.

\section{Summary}

In this work, three types of internal kink modes (an electron saturated internal kink mode, an electron resonant internal kink mode (RK), and a double efishbone) excited by energetic particles during ECRH/ECCD heating have been studied by the 24 $($ poloidal $) \times 16($ radial $)=384$ channel ECEI system on the HL-2A tokamak. These modes have been found in the low density discharges and their frequencies are close to the precessional frequency of the trapped fast electrons. Their mode numbers and mode structures have been identified by the newly developed ECEI system. The frequency of e-SK is nearly unchanged, while the e-RK shows rapid frequency chirping. The e-SK and e-RK rotate in the electron diamagnetic direction poloidally and are destabilized by the energetic trapped electrons. We found that there is a $q_{\min }$ threshold for the destabilization of e-SK. The e-SK is destabilized in the case of $q_{\min }>1$, while the RK is destabilized in the case of $q_{\min }<1$. The double e-fishbone has two $\mathrm{m} / \mathrm{n}=1 / 1$ modes which propagate in the opposite directions poloidally. The two modes are excited in a weak/negative magnetic central shear configuration, which is achieved with localized ECRH/ECCD during plasma current ramp-up. Strong thermal transfer and mode-mode coupling between the two $m / n=1 / 1$ modes have been observed, which seems to be related to the nonlinear interaction between the two modes and local heat transport during the MHD activity.

This work is supported by Chinese National Fusion Project for ITER under Grant No.2013GB104000, 2014GB107001 and 2013GB107002, and National Natural Science Foundation of China (11275062, 11475057, 11405049, 11475058, 11005037).

\section{References}

1. A. Fasoli, et.al., Nucl. Fusion 47, S264 (2007)

2. K. McGuire, et.al., Phys. Rev. Lett.50, 891 (1983)

3. W. Chen, et.al., Nucl. Fusion 50, 084008 (2010) 
4. X. T. Ding, et.al., Nucl. Fusion 42, 491 (2002)

5. L. W. Yan, et.al., Chin. Phys. Lett. 18, 1227 (2001)

6. Macor, et.al., Phys. Rev. Lett. 102, 155005 (2009)

7. L. M. Yu, et. al., Nucl. Fusion 53, 053002(2013)

8. F.Nanais, et.al., Nucl. Fusion 45, 102509 (2005)

9. L. Chen, et. al., Phys. Rev. Lett. 52, 1122 (1984)

10. R. Betti, et.al., Phys. Rev. Lett. 70, 3428 (1993)

11. F. Wang, et.al., Phys. of Plasmas 20, 102506 (2012)

12. I.T. Chapman, et.al., Nucl. Fusion 51, 073040 (2011)

13. J.E. Menard, et.al., Nucl. Fusion 45, 539 (2005)

14. W. Chen, et.al., Nucl. Fusion 49, 075022 (2009)

15. W. Deng, et.al., Nucl. Fusion 54, 013010 (2014)

16. X.R. Duan, et. al., Nucl. Fusion 53, 104009 (2013)

17. Equipe TFR, Nucl. Fusion 18, 647 (1978)

18. Y. Zhou, et.al., Rev. Sci. Instrum. 83, $10 \mathrm{E} 336$ (2012)

19. W.L. Zhong, et.al., Rev. Sci. Instrum. 85, 013507 (2014)

20. Z. B. Shi, et.al., Rev. Sci. Instrum. 85, 023510 (2014)

21. M. Bornatici, et.al., 1978 Nucl. Fusion 23, 1153 (1983)

22. M. Jiang, et.al., Rev. Sci. Instrum. 84, 113501 (2013)

23. L. M.Yu, et.al., Nucl. Fusion, 57, 036023 (2017)

24. T. Chapman, et.al., Nucl. Fusion 50, 045007 (2010)

25. R. B. Zhang, et. al., Plasma Phys. Control. Fusion 56, 095007 ( 2014)

26. M. Jiang, et.al., Phys. Plasmas, 24, 022110 (2017)

27. K.Matsuda, et.al., IEEE Trans. Plasma Sci. 17, 6 (1989) 